\title{
Pancreatic intraductal tubulopapillary neoplasm with associated invasive cancer successfully treated by total pancreatectomy: A case report
}

\author{
YUJI FUJIMOTO ${ }^{1}$, YOSHITO TOMIMARU ${ }^{1}$, HIROMI TAMURA ${ }^{2}$, KOZO NOGUCHI $^{1}$, \\ HIROTSUGU NAGASE ${ }^{1}$, ATSUSHI HAMABE ${ }^{1}$, MASASHI HIROTA ${ }^{1}$, KAZUTERU OSHIMA ${ }^{1}$, \\ TSUKASA TANIDA $^{1}$, TOMONO KAWASE ${ }^{1}$, SHUNJI MORITA ${ }^{1}$, HIROSHI IMAMURA ${ }^{1}$, TAKASHI IWAZAWA ${ }^{1}$, \\ KENZO AKAGI $^{1}$, MASASHI YAMAMOTO ${ }^{3}$, TSUTOMU NISHIDA $^{3}$, SHIRO ADACHI $^{2}$ and KEIZO DONO ${ }^{1}$
}

Departments of ${ }^{1}$ Surgery, ${ }^{2}$ Pathology and ${ }^{3}$ Gastroenterology, Toyonaka Municipal Hospital, Toyonaka, Osaka 560-8565, Japan

Received June 5, 2016; Accepted March 24, 2017

DOI: $10.3892 / \mathrm{ol} .2017 .6130$

\begin{abstract}
A 74-year-old male was admitted to Departments of Surgery, Toyonaka Municipal Hospital (Osaka, Japan) for treatment of a pancreatic tumor. Contrast enhanced computed tomography (CT) revealed a mass with small cystic lesions in the pancreatic head and body. Fluorodeoxyglucose-positron emission tomography/CT revealed an abnormal uptake of fluorodeoxyglucose, corresponding to the mass lesions. Upper gastrointestinal endoscopy revealed rough mucosa near the opening of the accessory pancreatic duct, and the mucosa biopsy exhibited adenocarcinoma with no mucin observed. The preoperative diagnosis was pancreatic intraductal tubulopapillary neoplasm (ITPN) with cancerous lesions, and a total pancreatectomy with splenectomy was performed. The resected tissue specimen revealed a solid tumor occupying the entire pancreas with intraductal growth into the main pancreatic duct. Histological examination revealed high-grade dysplastic cells in a tubulopapillary growth pattern without overt mucin production beyond the pancreatic duct. Immunohistochemical staining analysis of the tumor was positive for cytokeratin (CK)7, CK19 and mucin (MUC)1, and negative for MUC2, MUC5AC, MUC6 and caudal type homeobox 2. The tumor was finally diagnosed as pancreatic ITPN with associated invasive cancer. The patient remains well without evident recurrence nine months post-surgery. ITPN is a rare type of epithelial neoplasm of the pancreas, and is characterized by intraductal tubulo-papillary growth, ductal differentiation, limited intracellular mucin production, and cellular dysplasia. The present case report may contribute to improved understanding of how to effectively treat patients with ITPN.
\end{abstract}

Correspondence to: Dr Yoshito Tomimaru, Department of Surgery, Toyonaka Municipal Hospital, 4-14-1 Shibahara, Toyonaka, Osaka 560-8565, Japan

E-mail: ytomimaru@gmail.com

Key words: intraductal tubulopapillary neoplasm, intraductal neoplasm, pancreatic cancer, pancreas

\section{Introduction}

Intraductal tubulopapillary neoplasm (ITPN) is a rare type of epithelial neoplasm of the pancreas that is characterized by an intraductal, grossly visible, tubule-forming epithelial neoplasm with cellular dysplasia and ductal differentiation without overt mucin production (1). To the best of our knowledge, ITPN-like intraductal neoplasm was first recognized by Japanese investigators in the mid 1990s and was studied in 2009 by Yamaguchi et al (2). Following these reports, ITPN was adopted by the World Health Organization (WHO) classification, which was revised in 2010, as a subclass of intraductal neoplasms of the pancreas, along with intraductal papillary mucinous neoplasm (IPMN). It is estimated that ITPNs account for $<1 \%$ of all pancreatic exocrine tumor cases and 3\% of all pancreatic intraductal neoplasm cases (2). Due to the rarity of ITPN, information regarding the disease is currently limited, and only a few reports, case series and reviews are available $(2,3)$; thus, the clinicopathological features of ITPN remain to be elucidated. In this context, even a case report of ITPN is essential for further characterizing this disease in order to improve the management and treatment of patients with ITPN. In this report we present a case of pancreatic ITPN with associated invasive cancer that was successfully treated with total pancreatectomy.

\section{Case Report}

A 74-year-old male was admitted to the Departments of Surgery, Toyonaka Municipal Hospital (Osaka, Japan) for treatment of a pancreatic tumor. The patient's medical history included alcoholic acute pancreatitis, a renal stone and cerebral infarction. The patient did not exhibit any significant findings on physical examination. The laboratory analysis results were within the normal range, with the exception of the serum glucose level $(155 \mathrm{mg} / \mathrm{dl}$; normal range $60-110 \mathrm{mg} / \mathrm{dl})$ and HbAlc-NGSP (7.0\%; normal range 4.6-6.2\%), which were elevated. The levels of various tumor markers were within the normal range, including carcinoembryonic antigen $(2.8 \mathrm{ng} / \mathrm{ml}$; normal range, $<5.0 \mathrm{mg} / \mathrm{dl})$, cancer antigen $19-9(15 \mathrm{U} / \mathrm{ml}$; normal range, $<37 \mathrm{U} / \mathrm{ml})$, s-pancreas-1 antigen $(8.6 \mathrm{U} / \mathrm{ml}$; normal range, 

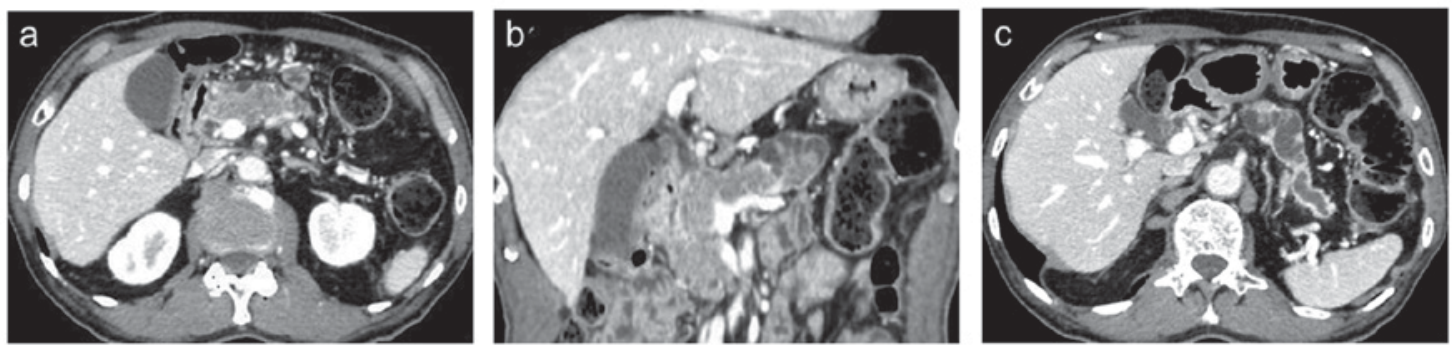

Figure 1. Preoperative radiological imaging using enhanced abdominal CT. (A and B) CT images revealed a mass with small cystic lesions in the pancreatic head and body (A, axial scan; B, coronal scan). (C) The main pancreatic duct at the peripheral side of the mass was dilated to $18 \mathrm{~mm}$. CT, computed tomography.
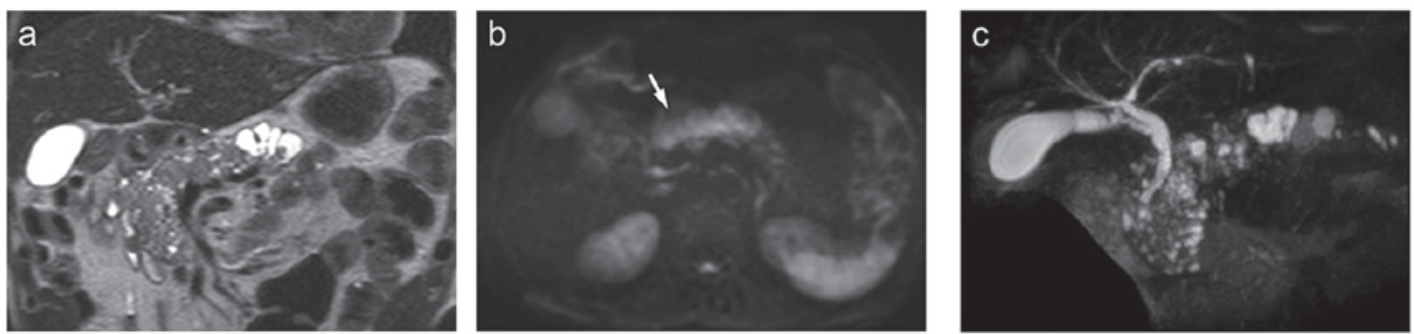

Figure 2. Preoperative radiological imaging by MRI. (A) A mass with small cystic lesions was identified on T2-weighted images (coronal scan). (B) The mass in the pancreatic head and body was visualized using high signal intensity on diffusion weighted images (arrow). (C) MRCP revealed small cystic lesions in the mass and a dilatation of the main pancreatic duct from the pancreatic body to the tail. MRI, magnetic resonance imaging; MRCP, magnetic resonance cholangiopancreatography.
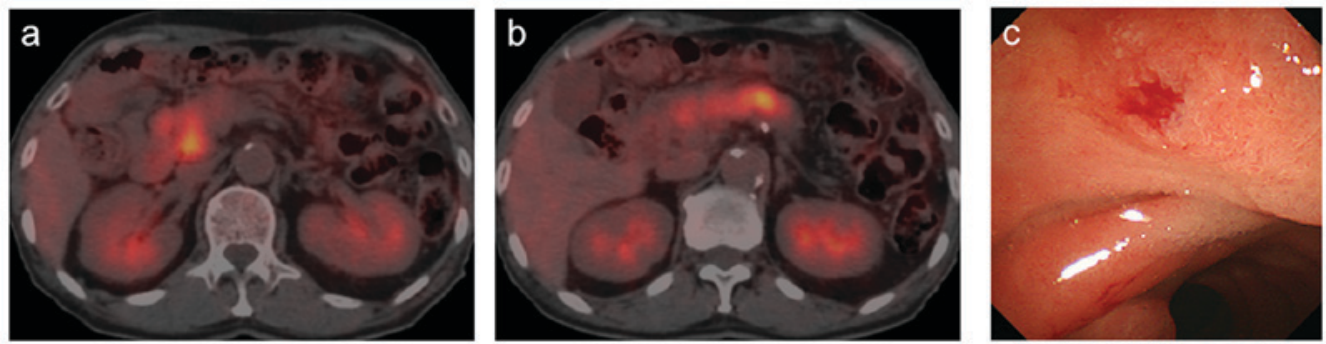

Figure 3. Findings of the FDG-PET/CT and upper gastrointestinal endoscopy. (A and B) Coronal scan of FDG-PET/CT revealed abnormal FDG uptake with a standardized uptake value maximum of 4.9 for the mass. (C) Upper gastrointestinal endoscopy revealed rough mucosa near the opening of the accessory pancreatic duct, and a biopsy from the mucosa indicated adenocarcinoma. No mucus was identified during this examination. FDG, 18F-fluorodeoxyglucose; PET, positron emission tomography; CT, computed tomography.

$<30 \mathrm{U} / \mathrm{ml}$ ) and duke pancreatic monoclonal antigen type 2 ( $46 \mathrm{U} / \mathrm{ml}$; normal range, $<150 \mathrm{U} / \mathrm{ml}$ ), and the serum IgG4 level was also normal $(42.8 \mathrm{mg} / \mathrm{dl}$; normal range, $4.8-105 \mathrm{mg} / \mathrm{dl})$. Contrast-enhanced computed tomography (CT) revealed a mass with small cystic lesions in the pancreatic head and body that exhibited a non-uniform contrast effect (Fig. 1A and B). The main pancreatic duct at the peripheral side of the mass was dilated to $18 \mathrm{~mm}$ (Fig. 1C). Although the patient was not jaundiced, the lower common bile duct was surrounded by the mass, which was in contact with the portal vein and the superior mesenteric vein. There were no visibly enlarged lymph nodes. Magnetic resonance imaging (MRI), as with CT, revealed small cystic lesions in the mass on T2-weighted images (Fig. 2A). The mass in the pancreatic head and body was visualized with high signal intensity on diffusion-weighted images (Fig. 2B). On MR cholangiopancreatography (MRCP), there were small cystic lesions present in the mass and dilatation of the main pancreatic duct from the pancreatic body to the tail (Fig. 2C). Upper gastrointestinal endoscopy revealed rough mucosa near the opening of the accessory pancreatic duct and no mucus was observed (Fig. 3A). Biopsy of the mucosa revealed adenocarcinoma. An 18F-fluorodeoxyglucose (FDG)-positron emission tomography scan revealed abnormal FDG uptake with a maximum standardized uptake value of 4.9 for the mass (Fig. 3B). Based on the aforementioned findings, the pre-operative diagnosis was pancreatic ITPN with associated cancer lesions. Although IPMN was also considered as another possible differential diagnosis of the mass, this diagnosis was rejected due to the lack of mucous secretion identified. A laparotomy using an upper and middle abdominal median incision was performed under general anesthesia. The whole pancreas was hard, likely due to the patient's previous pancreatitis. As the mass was located in the entire pancreatic head and body, an attempt was made to resect the pancreas on the tail side of the mass, in order to preserve the pancreatic tail. However, it was problematic to separate the pancreatic body and the splenic artery and vein, due to the tissue hardness. Therefore, it was judged to be impossible to preserve the spleen, and a total pancreatectomy with splenectomy was 

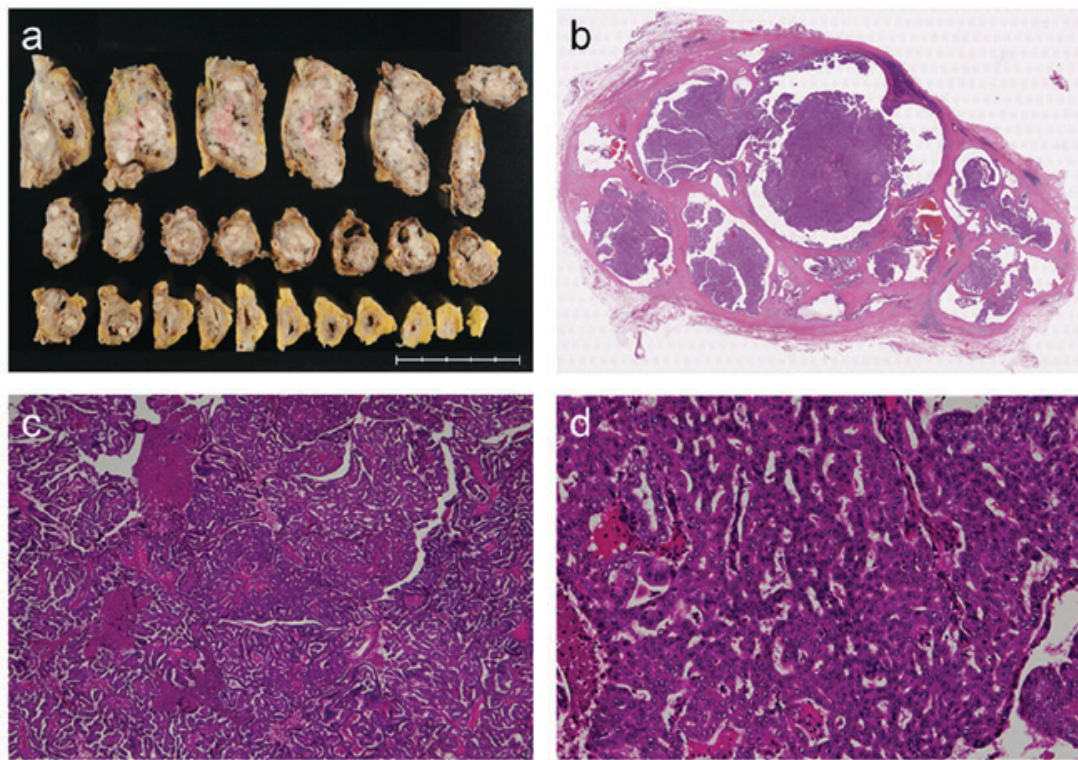

Figure 4. Macroscopic and microscopic findings of the resected tissue specimen. (A) The whole pancreas was hard, and the solid tumor occupied the entire pancreas. (B) In the macroscopic view of the pancreas stained with hematoxylin and eosin, the tumor was revealed to be occupying the entire pancreas with intraductal growth of the main pancreatic duct. (C and D) Histological analysis of the tumor tissue revealed high-grade dysplastic cells in a tubulopapillary growth pattern without the overt production of mucin (C, low-power field; D, high-power field).
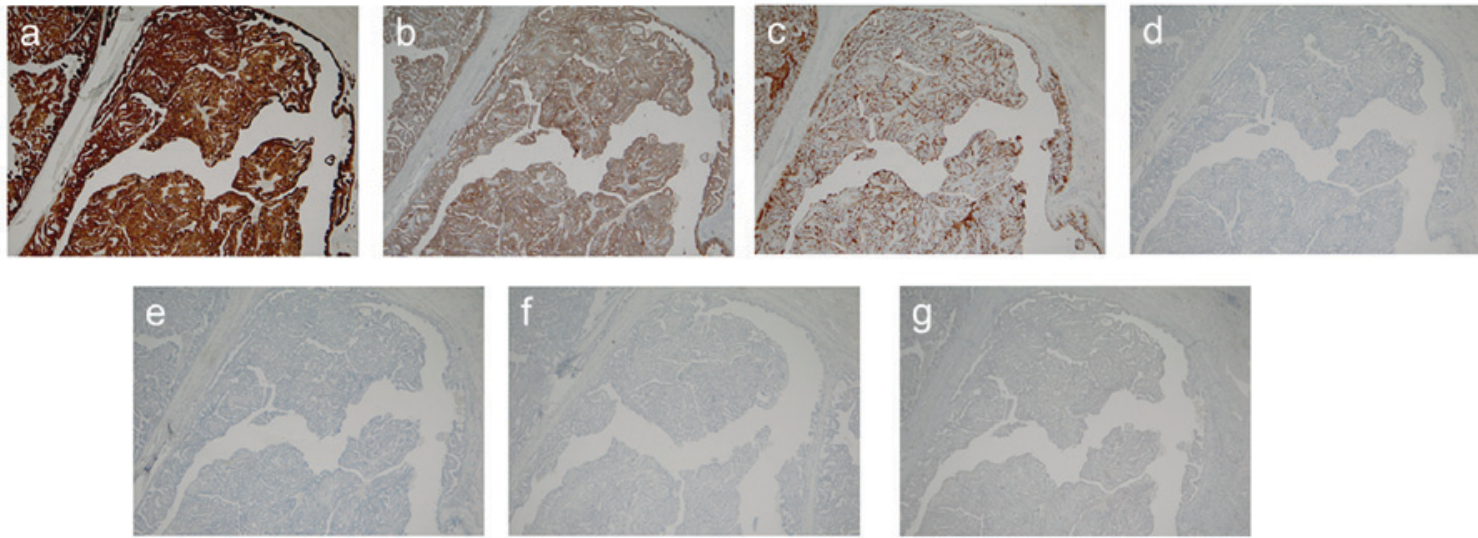

Figure 5. Immunohistochemical staining of the tumor. The tumor was immunohistochemically stained for (A) CK7, (B) CK19, (C) MUC1, (D) MUC2, (E) MUC5AC, (F) MUC6 and (G) CDX2. The staining was positive for CK7, CK19 and MUC1, and negative for MUC2, MUC5AC, MUC6 and CDX2. CK, cytokeratin; MUC, mucin; CDX2, caudal type homeobox 2.

subsequently performed. Lymphadenectomy was performed for dissecting regional lymph nodes. Macroscopic examination of the resected specimen indicated an off-white solid tumor occupying the entire pancreas with intraductal growth of the main pancreatic duct; mucin was not identified (Fig. 4A and B). Histological examination using hematoxylin and eosin staining revelaed that the tumor exhibited high-grade dysplastic cells in a tubulopapillary growth pattern without the overt production of mucin (Fig. 4C and D). The tumor had infiltrated the main pancreatic duct, although the pre-operative CT scan had not revealed any tumors in the main pancreatic duct of the pancreatic tail. The tumor had invaded beyond this to the entire pancreatic parenchyma and serosal invasion and retroperitoneal invasion were observed, whereas vascular invasion was not identified. Among 30 lymph nodes dissected, metastasis was verified to be present in two lymph nodes. The metastases were also identified in the lymph nodes along the common hepatic artery and the splenic artery. No cancer cells were identified in the resected cut end margin of bile duct or dissected peripancreatic tissue. Immunohistochemical staining was positive for cytokeratin (CK)7 (Roche Diagnostics, Basel, Switzerland), CK19 (Leica Microsystems, Ltd., Milton Keynes, UK) and mucin (MUC1) (Leica Microsystems Ltd.), and negative for MUC2 (Leica Microsystems, Ltd.), MUC5AC (Leica Microsystems, Ltd.), MUC6 (Leica Microsystems, Ltd.) and caudal type homeobox 2 (Biocare Medical, LLC., Concord, CA, USA; Fig. 5). The final diagnosis was determined to be pancreatic ITPN with associated invasive cancer. The patient progressed without post-operative complications. Following the surgery, the serum glucose levels were managed with subcutaneous insulin injections. At the time of this report (9 months post-surgery), the patient remains disease-free without evidence of recurrence, and is being followed on an outpatient basis (follow-up is ongoing for 5-years). 


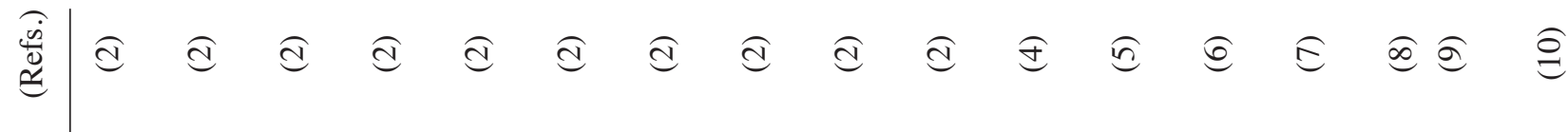

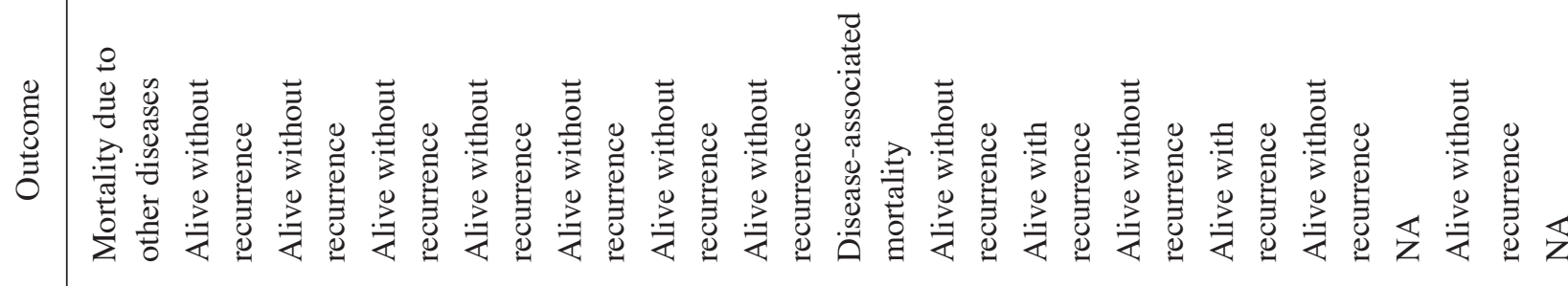
胥

苛 2.:

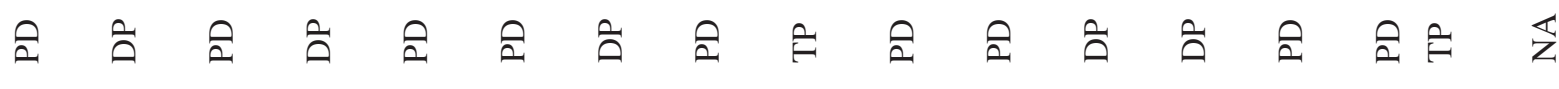

总

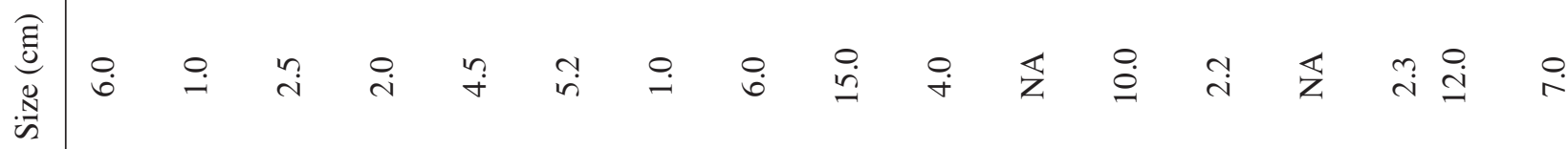

.ृ.气

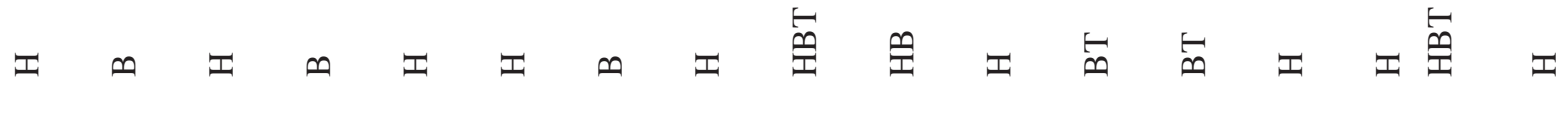

홀

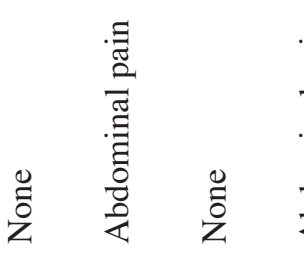

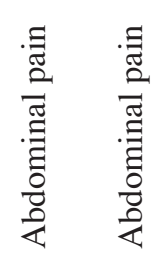

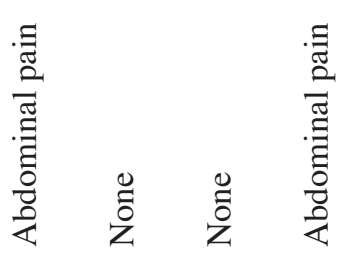

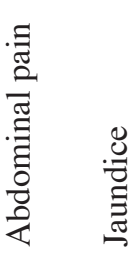
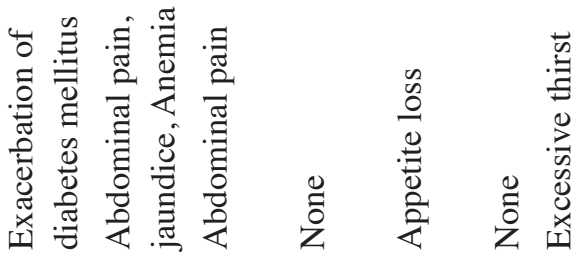

$\underset{\frac{\pi}{\tilde{g}}}{\grave{0}}$

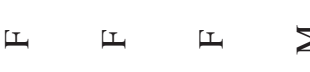

工

工

$\Sigma$

$\Sigma$

$\Sigma$

$\Sigma \Sigma$

\& $8 \backsim \infty$

$\therefore \sqrt{12}$

$\forall \quad$

$\stackrel{\infty}{\stackrel{\circ}{\vdash}}$

in

in

$\infty$

ঠ

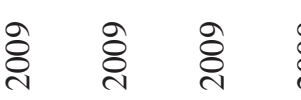

ठे ஓे

ठें छे छे

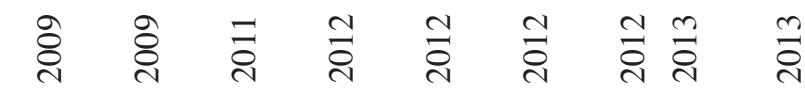

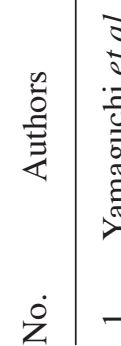

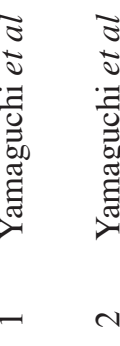

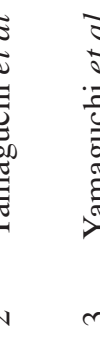
'

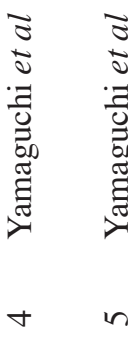

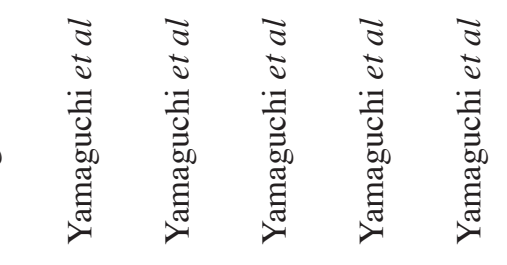

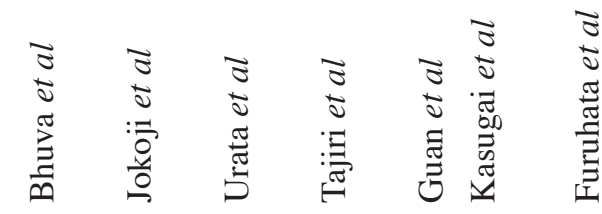

$\therefore=\simeq$

$9 \pm \cong$ 


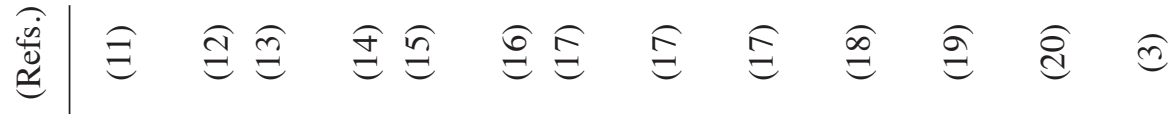

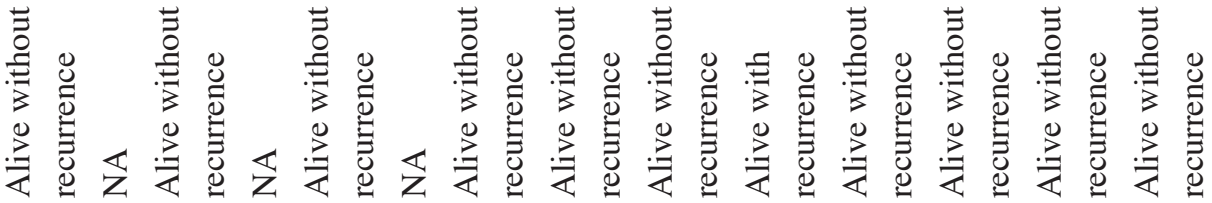

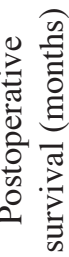

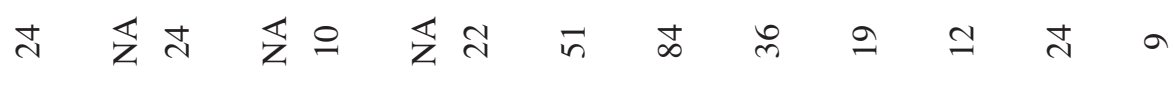

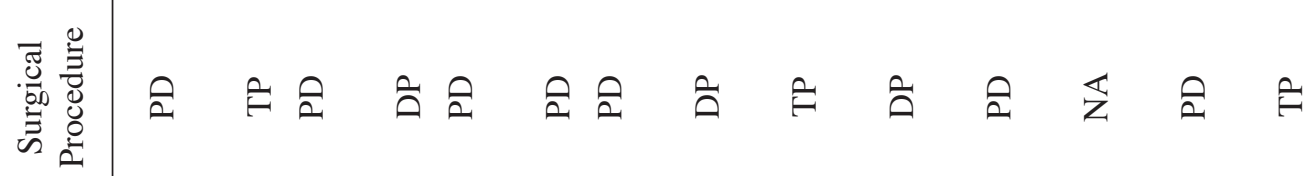

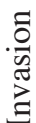

द्ध

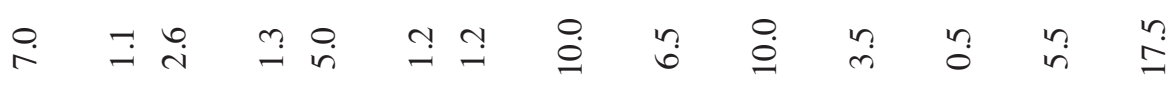

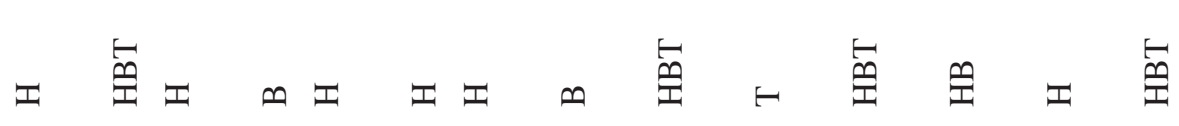

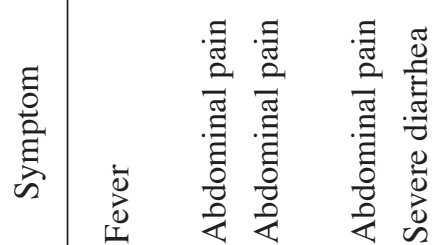

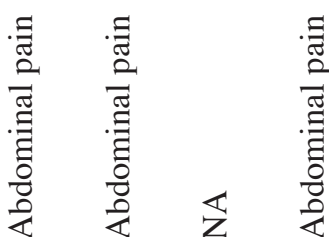

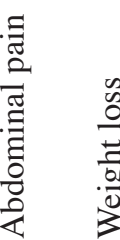

离

$\Sigma \quad \Sigma$

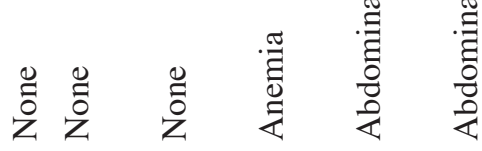

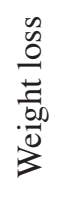

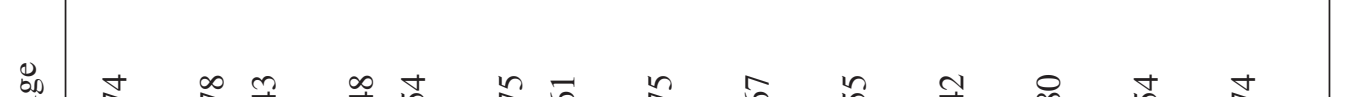

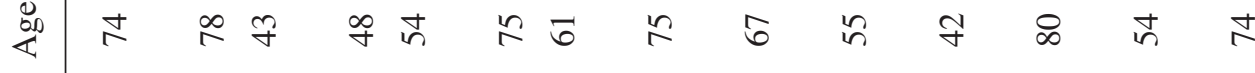

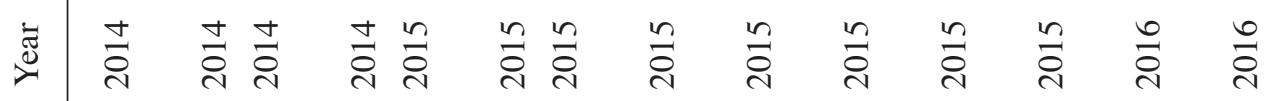

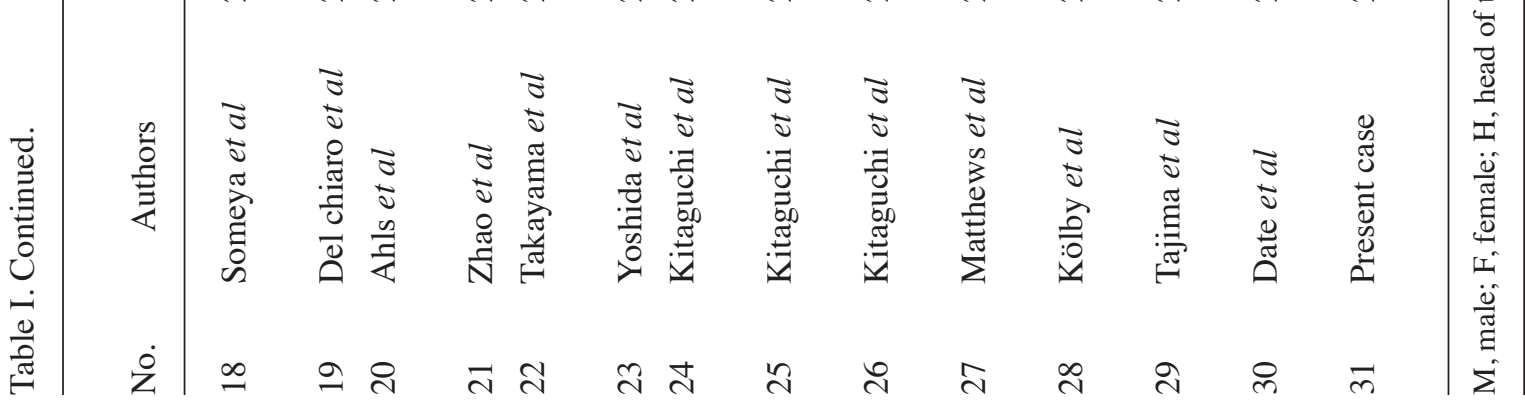




\section{Discussion}

Yamaguchi et al (2) reported 10 cases of pancreatic intraductal neoplasms with predominantly tubular growth patterns and a papillary component, and determined the neoplasm to be ITPN of the pancreas. To the best of our knowledge, that was the first report of ITPN. Intraductal neoplasms were classified as an IPMN or ITPN in the $2010 \mathrm{WHO}$ classification (1). ITPN is rare, accounting for $<1 \%$ of all pancreatic exocrine neoplasms and, to the best of our knowledge, there has been only one case series of patients with ITPN since the initial report by Yamaguchi et al (2). Date et al (3) recently analyzed the published data of 58 cases of ITPN, including their own case. In this study, they searched MEDLINE and Igakuchuo-Zacchi (a database of Japanese articles with English abstracts) for cases since 1980. The term ITPN was first introduced by Yamaguchi et al (2) in 2009; although, cases reported prior to 2009 were included in the study. This suggests that the diagnosis of ITPN in the cases reported prior to the definition may not be accurate, as the authors noted in the report. Therefore, in the present study, ITPN cases that had been reported in detail following the definition in 2009 were searched for, and only cases where the term ITPN was stated in the diagnosis were extracted. Overall, 30 cases were extracted (2,3-20). The clinicopathological features of 31 cases, including the extracted 30 cases and the current case, are presented in Table I. Among these 31 cases, 19 and 12 occurred in men and women, respectively. The age range of the patients involved was $35-80$ years, with a median age at diagnosis of 66 years. The most frequently reported symptom was abdominal pain, but there were also asymptomatic cases. Among the 31 patients, 29 patients had received surgery. The surgical procedure was pancreaticoduodenectomy in 16 patients, distal pancreatectomy in 8 patients and total pancreatectomy in 5 patients. Postoperatively, the overall 1-, 3- and 5-year survival rates were all 92.3\%. The summary of the clinicopathological features of the 31 cases is similar to that reported by Date et al (3). In addition, regardless of the presence of the invasive component in the ITPN area, all of the cases had associated cancer lesions. Therefore, all the cases were intraductal tubulopapillary cancer with or without an invasive component. There were no cases with intraductal tubulopapillary adenoma. This finding suggests ITPN cases are not similar to IPMN cases. The 2010 WHO classification categorizes IPMN cases according to their malignant transformation into IPMN with low or intermediate dysplasia, IPMN with high-grade dysplasia and IPMN with invasive cancer (1). One limitation was that a dedicated pathologist did not perform the histopathological diagnosis in the 31 cases; thus, this characteristic of ITPN must be validated in further and larger studies. In conclusion, the current study presents a case of ITPN with associated invasive cancer successfully treated with total pancreatectomy. Further characterization of ITPN based on a collection of cases, similar to that reported here, may lead to improved management of this type of neoplasm.

\section{References}

1. Adsay NV, Fukushima N, Furukawa T, Hruban RH, Klimstra DS Klöppel G, et al: Intraductal neoplasms of the pancreas. In: World Health Organization Classification of Tumours of the Digestive System. Bosman FT, Carneiro F, Hruban RH, Theise ND (eds). 4th edition. IARC, Lyon, France, pp304-313, 2010.
2. Yamaguchi H, Shimizu M, Ban S, Koyama I, Hatori T, Fujita I, Yamamoto M, Kawamura S, Kobayashi M, Ishida K, et al: Intraductal tubulopapillary neoplasms of the pancreas distinct from pancreatic intraepithelial neoplasia and intraductal papillary mucinous neoplasms. Am J Surg Pathol 33: 1164-1172, 2009.

3. Date K, Okabayashi T, Shima Y, Iwata J, Sumiyoshi T, Kozuki A, Morita S, Hata Y, Noda Y, Nishioka A and Matsumoto M: Clinicopathological features and surgical outcomes of intraductal tubulopapillary neoplasm of the pancreas: A systematic review. Langenbecks Arch Surg 401: 439-447, 2016.

4. Bhuva N, Wasan H, Spalding D, Stamp G and Harrison M: Intraductal tubulopapillary neoplasm of the pancreas as a radiation induced malignancy. BMJ Case Rep pii: bcr0920114777, 2011

5. Jokoji R, Tsuji H, Tsujimoto M, Shinno N and Tori M: Intraductal tubulopapillary neoplasm of pancreas with stromal osseous and cartilaginous metaplasia; a case report. Pathol Int 62: 339-343, 2012.

6. Urata T, Naito Y, Nagamine M, Izumi Y, Tonaki G, Iwasaki H, Sasaki A, Yamasaki A, Minami N, Yoshioka R, et al: Intraductal tubulopapillary neoplasm of the pancreas with somatic BRAF mutation. Clin J Gastroenterol 5: 413-420, 2012.

7. Tajiri T, Tate G, Matsumoto K, Hoshino H, Iwamura $T$, Kodaira Y, Takahashi K, Ohike N, Kunimura T, Mitsuya T and Morohoshi T: Diagnostic challenge: Intraductal neoplasms of the pancreatobiliary system. Pathol Res Pract 208: 691-696, 2012.

8. Guan H, Gurda G, Lennon AM, Hruban RH and Erozan YS: Intraductal tubulopapillary neoplasm of the pancreas on fine needle aspiration: Case report with differential diagnosis. Diagn Cytopathol 42: 156-160, 2014.

9. Kasugai H, Tajiri T, Takehara Y, Mukai S, Tanaka J and Kudo SE: Intraductal tubulopapillary neoplasms of the pancreas: Case report and review of the literature. J Nippon Med Sch 80: 224-229, 2013.

10. Furuhata A, Minamiguchi S, Mikami Y, Kodama Y, Sumiyoshi S, Adachi $\mathrm{S}$ and Haga $\mathrm{H}$ : Intraductal tubulopapillary neoplasm with expansile invasive carcinoma of the pancreas diagnosed by endoscopic ultrasonography-guided fine needle aspiration: A case report. Diagn Cytopathol 42: 314-320, 2014.

11. Someya Y, Nakamoto Y, Nakatani K, Kawaguchi M, Minamiguchi S and Togashi K: 18F-FDG uptake in intraductal tubulopapillary neoplasm of the pancreas. Clin Nucl Med 39: e277-e280, 2014.

12. Del Chiaro M, Mucelli RP, Blomberg J, Segersvärd R and Verbeke C: Is intraductal tubulopapillary neoplasia a new entity in the spectrum of familial pancreatic cancer syndrome? Fam Cancer 13: 227-229, 2014.

13. Ahls MG, Niedergethmann M, Dinter D, Sauer C, Lüttges J, Post S, Marx A and Gaiser T: Case report: Intraductal tubulopapillary neoplasm of the pancreas with unique clear cell phenotype. Diagn Pathol 9: 11, 2014.

14. Zhao L, Hart J, Xiao SY and Antic T: Cytological features of pancreatic intraductal tubulopapillary neoplasm and an unexpected immunohistochemical profile. Pathology 46: 662-665, 2014.

15. Takayama S, Maeda T, Nishihara M, Kanazawa A, Chong HS, Oka H, Hirota S and Ishikawa O: A case of intraductal tubulopapillary neoplasm of pancreas with severe calcification, a potential pitfall in diagnostic imaging. Pathol Int 65: 501-506, 2015.

16. Yoshida Y, Matsubayashi H, Sasaki K, Kanemoto H, Uesaka K and Ono H: Intraductal tubulopapillary neoplasm of the pancreatic branch duct showing atypical images. J Dig Dis 16: 357-361, 2015.

17. Kitaguchi K, Kato Y, Kojima M, Okubo S, Takahashi D, Okada R, Nakayama Y, Nishida Y, Gotohda N, Takahashi S and Konishi M: A resected case of intraductal tubulopapillary neoplasm of the pancreas: Report of a case. Int Surg 100: 281-286, 2015.

18. Matthews Y, McKenzie C, Byrne C and Kench JG: Intraductal tubulopapillary neoplasm of pancreas with associated invasive carcinoma, lymph node, rectal and hepatic metastases. Pathology 47: 169-171, 2015.

19. Kölby D, Thilén J, Andersson R, Sasor A and Ansari D: Multifocal intraductal tubulopapillary neoplasm of the pancreas with total pancreatectomy: Report of a case and review of literature. Int J Clin Exp Pathol 8: 9672-9680, 2015.

20. Tajima S: Intraductal tubulopapillary neoplasm of the pancreas suspected by endoscopic ultrasonography-fine-needle aspiration cytology: Report of a case confirmed by surgical specimen histology. Diagn Cytopathol 43: 1003-1006, 2015. 